\section{Tolerating iPSCs}

Induced pluripotent stem cells (iPSCs) have great potential in regenerative medicine; however, the genetic reprogramming needed to produce these cells has left questions about their possible immunogenicity. In Nature, Abe and colleagues provide strong evidence suggesting that the ten IPSC lines they examine show minimal signs of immunogenicity. The authors use iPSCs derived from C57BL/6 mice to generate highly chimeric mice in which both the skin epithelium and bone marrow arise from differentiated iPSCs. After transplantion into syngeneic hosts, iPSC-derived skin or bone marrow shows little if any evidence of rejection and is essentially tolerated as well as embryonic stem cell-derived tissues. Bone marrow cells that arise from iPSCs can also reconstitute lethally irradiated hosts and thus seem to be functionally normal. These findings could allay fears about one of the main potential impediments to the clinical use of iPSCs. ZF Nature 494, 100-104 (2013)

\section{Function of the IgM receptor}

Toso is a plasma membrane protein expressed by many cell types of the immune system and acts as an Fc receptor for immunoglobulin $\mathrm{M}$ but can also inhibit proapoptotic signals. In the Proceedings of the National Academy of Sciences, Mak and colleagues demonstrate a further important function for this molecule in modulating phagocyte activation. The authors generate Toso-deficient mice and find no obvious defects in the development of their phagocytes; however, in response to various activators, Toso-deficient granulocytes produce a greater abundance of reactive oxygen species (ROS). Conversely, signaling via Toll-like receptors, production of inflammatory cytokines, phagocytosis of Listeria and sepsis induction are all diminished in Toso-deficient phagocytes. Finally, the authors find that the Toso-deficient mice have impaired resistance in a Listeria infection model. Toso therefore seems to exert dual functions in phagocytes by inhibiting programmed cell death but also diminishing their activation threshold to a variety of triggers, most probably in an immunoglobulin M-dependent manner.

Proc. Natl. Acad. Sci. USA 110, 2593-2598 (2013)

\section{ILC2 cells and metabolic homeostasis}

Eosinophils in the visceral adipose tissue (VAT) contribute to the maintenance of alternatively activated (M2) macrophages and insulin sensitivity. In The Journal of Experimental Medicine, Locksley and colleagues show that innate lymphoid type 2 cells (ILC2 cells) resident in the VAT promote the accumulation of eosinophils through the production of interleukin 5 (IL-5) and IL-13. ILC2 cells are the main producers of these cytokines in the VAT in the basal state or after IL-33-mediated activation. Deficiency in IL-5 or IL-13, depletion of ILC2 cells or consumption of a high-fat diet, which induces loss of adipose ILC2 cells, results in fewer eosinophils and M2 macrophages in the VAT, whereas helminth infection increases the number of eosinophils in the VAT in an ILC2 cell-dependent manner. These results indicate a role for ILC2 cells in metabolic homeostasis, probably through the maintenance of an anti-inflammatory state.

J. Exp. Med. (18 February 2013) doi:10.1084/jem.2012196

\section{Foxp3 phosphorylation}

Antagonism of tumor-necrosis factor (TNF) is an effective treatment for rheumatoid arthritis. In Nature Medicine, Zhang and colleagues show that TNF in the inflamed synovium of patients with rheumatoid arthritis induces dephosphorylation of a serine residue (Ser418) in the DNA-binding domain of the transcription factor Foxp3 and that phosphorylation at this site is required for the inhibitory function of regulatory $T$ cells $\left(T_{\text {reg }}\right.$ cells). The number of $T_{\text {reg }}$ cells is normal in the synovial fluid of patients with rheumatoid arthritis, but their suppressive function is impaired.

The authors show that TNF induces the upregulation of the phosphatase PP1 in $\mathrm{T}_{\text {reg }}$ cells, which interacts with Foxp3 and mediates dephosphorylation of Ser418. Treatment of rheumatoid arthritis patients with infliximab (antibody to TNF) restores both $\mathrm{T}_{\text {reg }}$ cell function and Foxp3 phosphorylation to levels similar to those in healthy subjects.

Nat. Med. (10 February 2013) doi:10.1038/nm.3085

Written by Laurie A. Dempsey, Zoltan Fehervari \& Ioana Visan

\section{LncRNA regulates Ifng}

Long noncoding RNA (IncRNA) is a recently discovered class of intergenic regulatory elements. In Cell, Gomez et al. report the functional activity of NeST, an enhancer-like IncRNA associated with interferon- $\gamma$ (IFN- $\gamma$ ) expression. NeST is encoded by a conserved gene adjacent to the Ifng locus; however, polymorphisms exist in mice that alter its expression, a trait that confers susceptibility to Theiler's virus but resistance to Salmonella infection. CD4 ${ }^{+} \mathrm{T}$ cells and natural killer cells have low expression of $\mathrm{NeST}$, but activated $\mathrm{CD} 8^{+} \mathrm{T}$ cells have higher expression of NeST. The IncRNA can function in either cis or trans to promote Ifng expression. NeST associates with WDR5, a component of the histone 3 lysine 4-methyltransferase complex that modifies the Ifng regulatory loci. NeST expression correlates with a greater abundance of trimethylated histone 3 lysine 4. Hence, NeST confers target specificity for epigenetic modification. It is unclear whether Ifng is the only target of NeST IncRNA; however, other examples of IncRNA-mediated gene regulation are sure to come. $L A D$ Cell 152, 743-754 (2013)

\section{Mitochondrial ROS}

ROS are known for their cytotoxic activities; however, increasing evidence points to ROS as a modifier of cell signaling. In Immunity, Sena et al. show that calcium-dependent mitochondrial production of ROS is necessary for antigen-driven activation of T cells. Mice whose T cells lack respiratory complex III and are thus unable to generate mitochondrial superoxide or $\mathrm{H}_{2} \mathrm{O}_{2}$ do not express IL-2 or upregulate expression of CD69 and CD25 in response to signaling via the T cell antigen receptor. Suprisingly, these mice have apparently normal thymopoiesis, and their peripheral T cells undergo homeostatic proliferation. Proximal $\mathrm{T}$ cell antigen receptor signals that lead to calcium influx are normal in T cells deficient in respiratory complex III. The authors trace the defect to a failure to translocate the transcription factor NFAT to the nucleus; however, the molecular targets sensitive to mitochondrial generation of ROS remain elusive.

Immunity 38, 225-236 (2013)
$L A D$ 